\title{
Quetiapine Ameliorates Anxiety-Like Behavior and Cognitive Impairments in Stressed Rats: Implications for the Treatment of Posttraumatic Stress Disorder
}

\author{
H.-N. WANG ${ }^{1 *}$, Y. PENG ${ }^{1 *}$, Q.-R. TAN ${ }^{1}$, Y.-C. CHEN ${ }^{1}$, R.-G. ZHANG ${ }^{1}$, Y.-T. QIAO ${ }^{1}$, \\ H.-H. WANG ${ }^{1}$, L. LIU' ${ }^{2}$, F. KUANG ${ }^{2}$, B.-R. WANG ${ }^{2}$, Z.-J. ZHANG ${ }^{3}$
}

*These authors contributed equally to this study

${ }^{1}$ Department of Psychiatry, Xijing Hospital, Fourth Military Medical University, Xi'an, Shaanxi, China, ${ }^{2}$ Institute of Neurosciences, Fourth Military Medical University, Xi'an, Shaanxi, China, ${ }^{3}$ Faculty of Medicine and School of Chinese Medicine, University of Hong Kong, Hong Kong, China

Received February 3, 2009

Accepted May 11, 2009

On-line June 19, 2009

\section{Summary}

The purpose of this study was to determine preventive and protective effects of chronic orally administration with quetiapine (QUE) against anxiety-like behavior and cognitive impairments in rats exposed to the enhanced single prolonged stress (ESPS), an animal model that is used to study post-traumatic stress disorder (PTSD), and to detect changes in the expression of cortical phosphorylated $\mathrm{p} 44 / 42$ extracellular-regulated protein kinase (pERK1/2). Before or after exposure to ESPS paradigm, consisting of 2-h constraint, 20-min forced swimming, etherinduced loss of consciousness, and an electric foot shock, rats were given orally QUE (10 mg/kg daily) for 14 days. Animals were then tested in the open field (OF), elevated plus-maze (EPM), and Morris water maze (MWM). Brains were removed for immunohistochemical staining of pERK1/2. ESPS exposure resulted in pronounced anxiety-like behavior compared to unexposed animals. ESPS-exposed animals also displayed marked learning and spatial memory impairments. However, QUE treatment (both before and after ESPS exposure) significantly ameliorated anxiety-like behavior, learning and spatial memory impairments. ESPS also markedly reduced the expression of PERK1/2 in the prefrontal cortex, medial amygdala nucleus, and cingulate gyrus. Both before and after ESPS exposure QUE treatments significantly elevated the reduced pERK1/2 expression in the three brain regions. QUE has preventive and protective effects against stress-associated symptoms and the changes in pERK1/2 functions may be associated with the pathophysiology of traumatic stress and the therapeutic efficacy of anti-PTSD therapy.

\section{Key words}

PTSD • Quetiapine • Anxiety • Cognitive impairment • ERK

\section{Corresponding author}

Q.-R. Tan, Department of Psychiatry, Xijing Hospital, Fourth Military Medical University, 17 Changle Road, Xi'an, Shaanxi 710032, China. Fax: (+86)-29-83293951. E-mail: tanqingr@fmmu.edu.cn

\section{Introduction}

Post-traumatic stress disorder (PTSD) is a group of symptoms that occur in individuals who have experienced an exposure to a dramatic stress. Cognitive deficits and memory dysfunction also frequently occur during the development of PTSD (Charles and Bremner 2006, Leskin et al. 2007, Rauch et al. 2008). Despite the fact that selective serotonin reuptake inhibitors (SSRIs) are the first choice in the treatment of PTSD and that meaningful treatment outcomes have been observed in the clinic practice, there have been several shortcomings of SSRIs, as evidenced by the limited efficacy and rather high remission rates (Davidson and Rasmussen 2006). 
Emerging evidence has shown that atypical antipsychotic agents can be used as augmentation therapy in PTSD patients with poor responses to antidepressants (Siddiqui et al. 2005, Gao et al. 2006). Several clinical studies have also observed the effectiveness of atypical antipsychotics in the treatment of PTSD patients with psychotic features (Pivac et al. 2006). Quetiapine (QUE) is a new-generation atypical antipsychotic drug widely used to treat schizophrenia and other psychotic disorders. It has been found to be effective in treating PTSD patients with comorbid psychosis (Hamner et al. 2003, Stathis et al. 2005, Ahearn et al. 2006). Given the effectiveness of QUE observed, it is believed that QUE is not only effective in treating PTSD patients with psychotic features, but may also be used in patients with PTSD. Since QUE possesses neuroprotective effects, it could also be effective in protecting against cognitive impairments (Luo et al. 2005, He et al. 2006).

It is well documented that the extracellular signal-regulated protein kinase (ERK), a member of the mitogen-activated protein kinase family (MAPK), is highly sensitive to stress and closely associated with cognitive and mood processing (Zheng et al. 2008). The inhibition of ERK pathway in the hippocampus and prefrontal cortex has been shown to cause anxiety-like and depressive-like behavior (Qi et al. 2009). On the other hand, atypical antipsychotic agents can be capable of inducing ERK phosphorylation in certain brain regions, including the prefrontal cortex (Luo et al. 2004, Browning et al. 2007), and in PC12 cells (Lu et al. 2005). These studies suggest that ERK may be involved in stress-associated pathophysiological processing and therapeutic effects of atypical antipsychotic drugs.

It is well established that physiological and behavioral changes observed in animals exposed to single prolonged stress (SPS) could appropriately represent pathophysiological process and core symptomatology of PTSD, including anxiety behavior and cognitive impairments (Liebsch et al. 1998, Takahashi et al. 2006, Iwamoto et al. 2007). SPS paradigms have been extensively applied in the investigation of PTSD. Our recent study has further shown that inescapable electric foot shock added to conventional SPS procedures significantly enhanced conditioned and sensitized fear responses (Wang et al. 2008). Moreover, early intervention with paroxetine, a selective serotonin reuptake inhibitor, effectively prevented the occurrence of PTSD-like behavior in this enhanced single prolonged stress (ESPS) procedures.
The present study sought to determine whether chronic treatment with QUE could ameliorate animals' stress-associated behaviors observed in ESPS paradigm, particularly anxiety-like behaviors and cognitive impairments. The study also aimed to detect the effects of ESPS exposure and QUE treatment on the expression of cortical phosphorylated $\mathrm{p} 44 / 42$ extracellular signalregulated protein kinase (pERK1/2) using immunohistochemical technique.

\section{Methods}

\section{Animals}

Adult male Sprague-Dawley rats (nearly eight weeks old) were used in the study. The study protocol was approved by the Committee of Animal Care and Use for Research and Education at the Fourth Military Medical University. Experiments were performed in accordance with the Guidelines for the Care and Use of Mammals in Neuroscience and Behavioral Research. Animals were housed in four per cage in an airconditioned room with a 12:12-h light/dark cycle, and free access to food and water. Animals were allowed to acclimate for at least 10 days before experiments.

\section{QUE treatment}

Quetiapine (AstraZeneca Pharmaceuticals, Macclesfield, UK) was dissolved in drinking water and applied via a lightproof bottle at doses of $10 \mathrm{mg} / \mathrm{kg}$ daily for 14 days before or after ESPS procedures (see below). Control animals received only tap water. The choice of the dose and the route of administration was based upon our preliminary experiments confirming no significant changes in body weight and the volume of water intake during the period in the current housing conditions, and the exclusion of additional stress potentially arisen from other routes (e.g. intraperitoneal or intragastric administration).

\section{Behavioral paradigms}

ESPS: Detailed ESPS procedure has been described in previous studies (Liberzon et al. 1997, 1999, Wang et al. 2008). Briefly, rats were restrained for $2 \mathrm{~h}$, immediately followed by forced swimming for $20 \mathrm{~min}$ in $24{ }^{\circ} \mathrm{C}$ water contained in a clear acrylic cylinder $(24 \mathrm{~cm}$ in diameter and $50 \mathrm{~cm}$ in height). After $15 \mathrm{~min}$ of recuperation, animals were exposed to diethyl ether until they lost consciousness, and then moved into a shock chamber. When they recovered (about $30 \mathrm{~min}$ ), a single 
electric foot shock ( $1 \mathrm{~mA}$ for $4 \mathrm{~s}$ ) was delivered via metal grids installed in the bottom of the chamber.

Open-field (OF) test: OF was used to test animals' spontaneous locomotor activity. The apparatus were composed of four same black acrylic plastic boxes which were placed in soundproof boxes. The acrylic box is formed a square area $(47 \times 47 \mathrm{~cm})$ with walls of $47 \mathrm{~cm}$ in height. The recording was performed in the soundproof box illuminated by a red fluorescent light $(30 \mathrm{~W})$. During testing, each rat was placed in the center zone at the beginning and horizontal distance traveled was automatically recorded for $15 \mathrm{~min}$ by an automatic analyzing system (DigBehav, Jiliang Co. Ltd, Shanghai, China). The total movement in the OF were analyzed. The test was carried out at 8:30 a.m. on the 15 th day after ESPS.

Elevated plus-maze (EPM) test: EPM has been well validated in detecting responses to external stressful stimuli (Liebsch et al. 1998). The Plexiglas apparatus consists of a plus-shaped platform elevated $50 \mathrm{~cm}$ above the floor. Two of the opposing arms $(50 \mathrm{~cm} \times 10 \mathrm{~cm})$ are enclosed by $40 \mathrm{~cm}$-high side and end walls (closed arms), other two arms were not installed with walls (open arms). At the beginning, animals were placed in the central area $(10 \times 10 \mathrm{~cm})$ of the maze, facing an enclosed arm. The exposure during initial $5 \mathrm{~min}$ was taped with a video camera. The following parameters were calculated by an investigator who was blind to treatment conditions of animals: percentages (\%) of both time spent and number of entry into open arms in reference to total time spent on all arms and total number of entries into all arms, respectively. The test was carried out one hour after OF test.

Morris water maze (MWM) test: Learning and spatial memory performance was measured using MWM based on the classic Morris protocol. MWM apparatus consisted of a black colored pool $(160 \mathrm{~cm}$ in diameter and $55 \mathrm{~cm}$ in height). The pool was housed in a temperaturecontrolled room and divided into four quadrants. For each experimental session, the pool was filled with $20-23{ }^{\circ} \mathrm{C}$, dark ink-stained water at $23 \mathrm{~cm}$ in height, and a cylindrical dark olive-green colored platform $(21 \mathrm{~cm}$ in height and $10 \mathrm{~cm}$ in diameter) was placed in one of the quadrants (the target quadrant). The platform was submerged approximately $2 \mathrm{~cm}$ below the surface of the water during the spatial learning trials. Three extra-maze cues were set on the wall surrounding the pool. A digital video camera was positioned directly above the pool enabling full collection of swim activity in the different quadrants and attached to a computer-controlled system (Jiliang software company, Shanghai, China).

Animals were initially brought into a quadrant (not containing the platform), with the head to face the wall. If animals could not find the escape platform within $60 \mathrm{~s}$, experimenters gently assisted animals onto the platform and allowed them to stay there for $20 \mathrm{~s}$. For learning performance, animals had four 60 -s learning trials daily each starting one of four quadrants in a random manner, with a 20 -s interval between trials. The escape latency to find the platform, calculated by averaging four trial values, was used to represent learning performance. Each animal was tested for consecutive five days for learning performance. Spatial memory was evaluated at day 6 using the probe test, i.e., the platform was removed and animals were placed at a novel position. Percentages of time spent in the target quadrant (platform removed) were calculated as an index for spatial memory.

\section{Immunohistochemical detection of pERK1/2}

On the 15 th day after ESPS, animals $(n=5$, for each group) were transaortically perfused with $100 \mathrm{ml}$ of saline, followed by $500 \mathrm{ml}$ of ice-cold $4 \%$ paraformaldehyde in $0.1 \mathrm{M}$ phosphate buffer (PB), $\mathrm{pH}$ 7.4. The forebrain was removed, placed in the same fixative for $4 \mathrm{~h}$ and transferred to $30 \%$ sucrose $(\mathrm{w} / \mathrm{v})$ in $0.1 \mathrm{M}$ PB for at least $24 \mathrm{~h}$ for cryoprotection. Immunohistochemical detection of pERK1/2 has been described in detail in our previous study (Wang et al. 2006). Briefly, following the completion of behavioral tests, animals were deeply anesthetized with pentobarbital (60 $\mathrm{mg} / \mathrm{kg}$, i.p.) and perfused transcardially with $4 \%$ paraformaldehyde. The brains were removed, fixed, and serially sectioned at $30 \mu \mathrm{m}$ in thickness. Every sixth sections were collected for immunohistochemical staining with rabbit anti-pERK1/2 primary antibody and avidin-biotin-peroxidase complex according to our standard staining protocol (Wang et al. 2006). pERK1/2labeled neuronal cells were counted under light microscope. Only those labeled cells with a sharply defined perimeter of the stained profile when focusing onto the surface of the section were counted. Since changes in pERK1/2 expression in the prefrontal cortex (PFC), medial amygdala nucleus (MAN), and cingulate gyrus (CG) have been well demonstrated under traumatic stress and atypical antipsychotic treatment (Luo et al. 2004, Browning et al. 2007, Qi et al. 2009), the three brain regions were brought to our central attention in the 


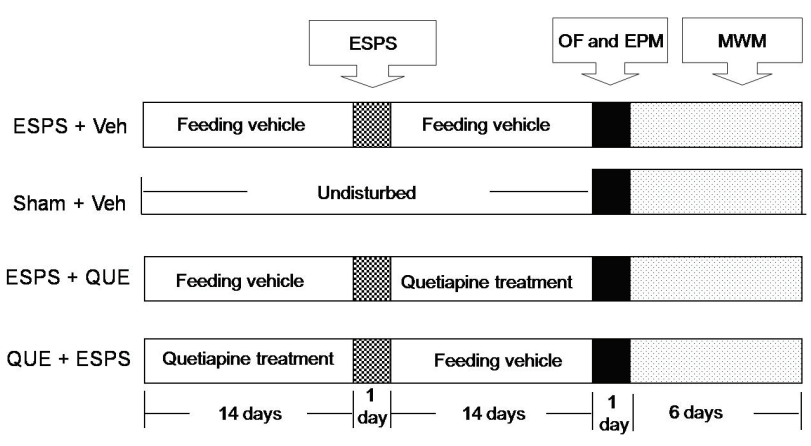

Fig. 1. Schematic diagram showing the experimental design, including the time duration or point for quetiapine/vehicle administration and the behavioral tests carried out in each group. Immunohistochemical detection was carried out at the fifteenth day after ESPS.

counting of pERK1/2-positive neurons. The definition of the three brain regions were based on the rat brain atlas of Paxinos and Watson (1998) and cell counts were conducted as described previously studies (Wang et al. 2006). The count results were expressed as the number of the cells per side (on the unilateral side) per section calculated by averaging across all sides of sections containing related brain regions.

\section{Experimental designs}

Chart flow of experimental procedures is shown in Figure 1. A total of 52 animals were used in the study. Following the acclimatization, animals were randomly assigned to one of four groups: (1) controls who were not exposed to ESPS but had vehicle treatment; (2) ESPS group who received vehicle treatment while underwent ESPS; (3) ESPS+QUE group who were treated with QUE after ESPS for 14 days; and (4) QUE+ESPS group who had 14-day QUE treatment before ESPS. Behavioral experiments started at a fixed time during testing days and animals were always habituated in the testing room for $15 \mathrm{~min}$ before behavioral tests. Each group was composed of 13 rats, 8 for behavioral tests and 5 for immunohistochemical staining.

\section{Statistical analysis}

Behavioral data (OF, EPM, and spatial memory index) and the number of pERK $1 / 2$ immunoreactive cells were analyzed using one-way analysis of variance (ANOVA) across the four groups. Learning performance data were analyzed using repeated measure two-way ANOVA (groups by days). Students-Newman-Keuls method was further used as post-hoc test to detect differences between groups. All data were expressed as means \pm S.E.M. All tests were two-sided and statistical
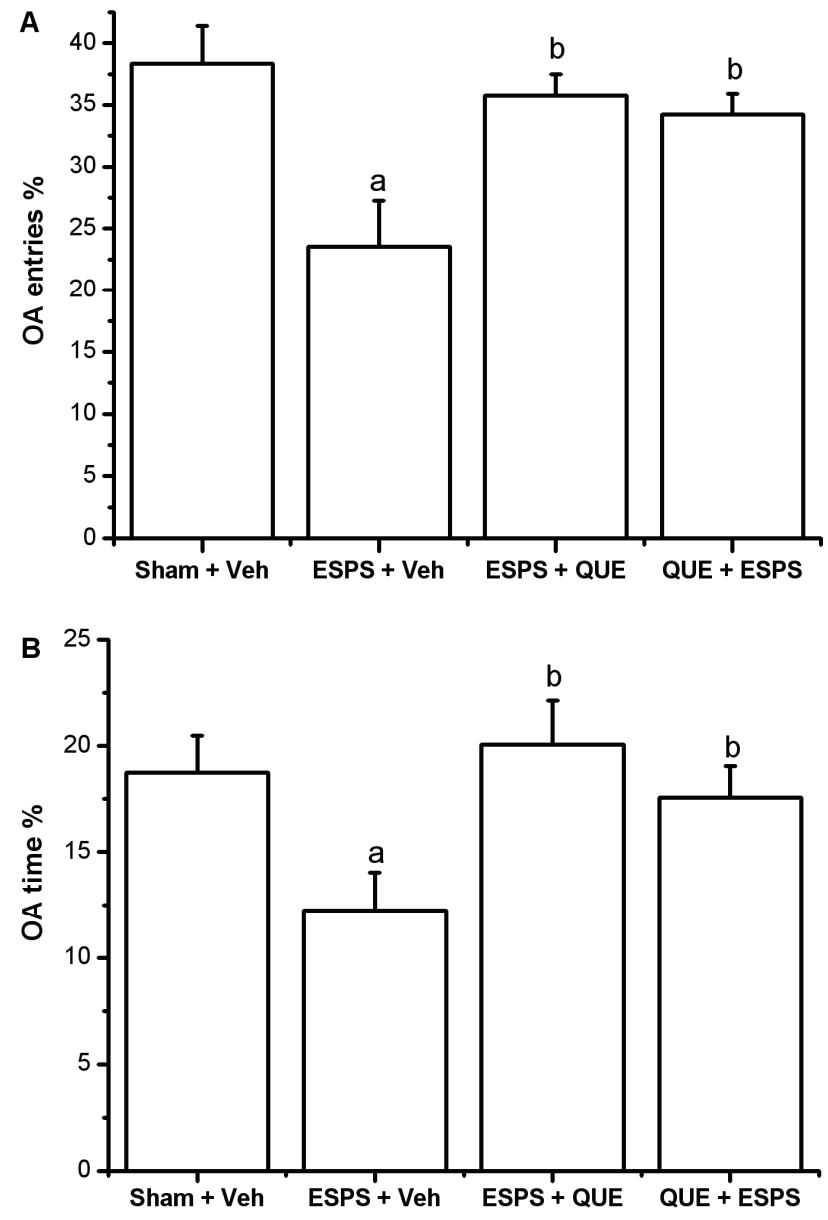

Fig. 2. Comparison of the percentage of open arm entries (open arm (OA) entries/total entries) (A) and the percentage of time spent in the open arms (B) in the EPM test among groups. a: Compared with Sham+Veh $(p<0.05)$, b: Compared with ESPS+Veh $(p<0.05)$.

significance was defined as $\mathrm{P}<0.05$.

\section{Results}

$O F$

One-way ANOVA analysis revealed no significant effect on the distance traveled across the four groups $\left(\mathrm{F}_{3,28}=1.262, \mathrm{P}=0.306\right)$ (data not shown).

\section{$E P M$}

The data of EPM test are illustrated in Figure 2. One-way ANOVA revealed significant effects of treatment on percentage of time spent $\left(\mathrm{F}_{3,28}=4.424\right.$, $\mathrm{P}=0.011)$ and percentage of the number of entry into open arms $\left(\mathrm{F}_{3,28}=3.584, \mathrm{P}=0.026\right)$. Between-group comparisons further showed that both EPM parameters were significantly decreased in ESPS-exposed rats compared to control animals $(\mathrm{P} \leq 0.025)$. QUE chronic treatment, both before and after the exposure to ESPS, 

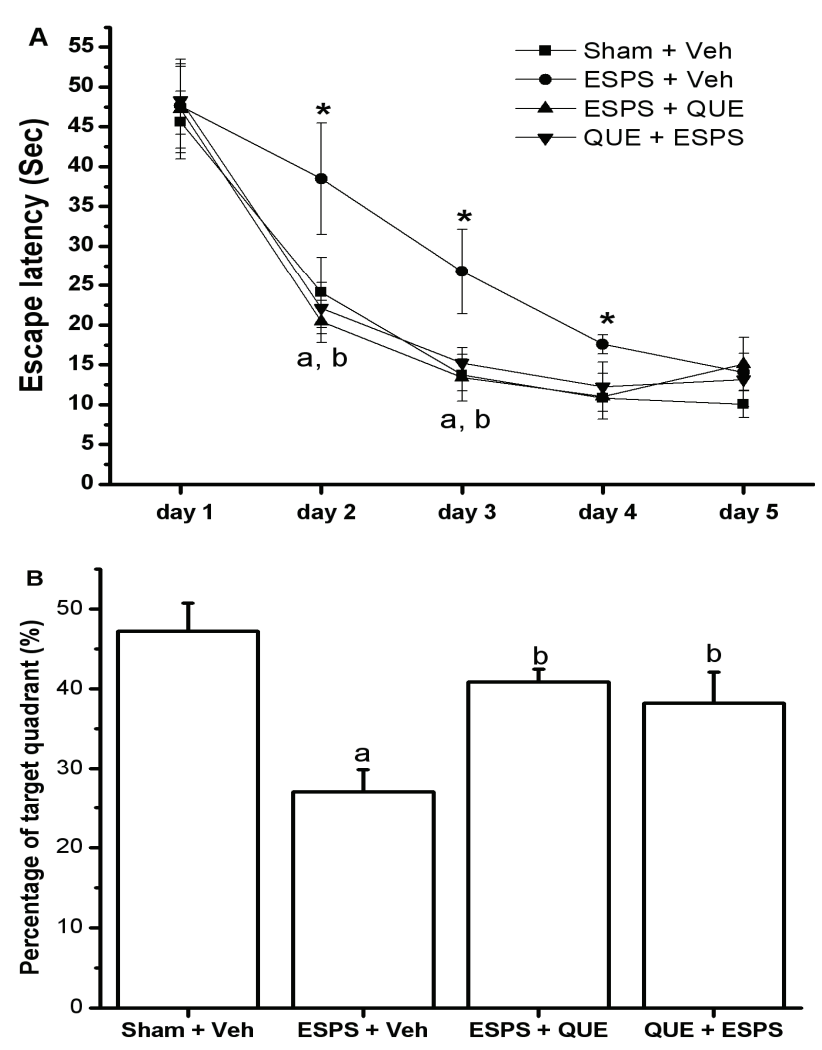

Fig. 3. Effects of ESPS and quetiapine on the mean escape latency (A), *: Compared with Sham+Veh $(p<0.05)$, a: QUE+ESPS was compared with ESPS+Veh $(p<0.05)$, b: ESPS+QUE was compared with ESPS+Veh $(p<0.05)$. Effects on the percentage of time spent in the target quadrant (B) in the MWM test. a: Compared with Sham+Veh $(p<0.05)$, b: Compared with ESPS+Veh $(p<0.05)$.

significantly reversed the decrease of the two EPM indices $(\mathrm{P} \leq 0.047)$.

\section{$M W M$}

Two-way ANOVA analysis revealed a significant interaction between groups and test days $\left(\mathrm{F}_{4,12}\right.$ $=3.884, \mathrm{P}<0.001)$ and significant main effects of groups $\left(\mathrm{F}_{3,28}=16.092, \mathrm{P}<0.001\right)$ and test days $\left(\mathrm{F}_{4,28}=286.044\right.$, $\mathrm{P}<0.001$ ) on the escape latency (Fig. 3A). Compared to control group, a striking increase of the latency was observed in ESPS-exposed animals at days 2 to 4 $(\mathrm{P} \leq 0.024)$. However, both groups of QUE-treated animals in pre- and post-exposure to ESPS had significantly shorter latency spent to find the underwater platform at day 2 and day 3 when compared with ESPS-exposed animals $(\mathrm{P}<0.001)$.

When the platform was removed from the pool at day 6 for spatial memory testing, a significant effect was observed across the four groups $\left(\mathrm{F}_{3,28}=36.813\right.$, $\mathrm{P}<0.001$, Fig. 3B). Between-group comparisons further showed that ESPS-exposed animals had spent much less time in target quadrant compared to control animals

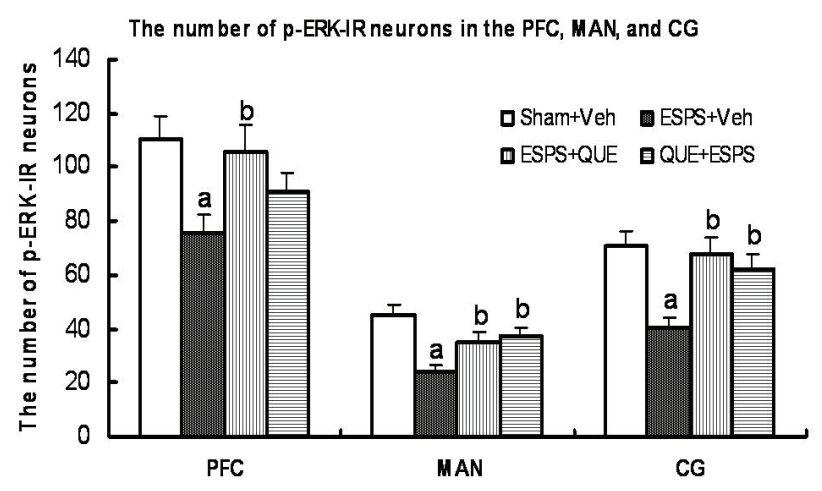

Fig. 4. Effects of ESPS and quetiapine on the pERK1/2 immunoreactive neuronal cells. Columns represent means \pm S.E.M. of the number of the cells per side (on the unilateral side) per section calculated by averaging across all sides of sections containing related brain regions. a: Compared with Sham+Veh $(p<0.05)$, b: Compared with ESPS+Veh $(p<0.05)$.
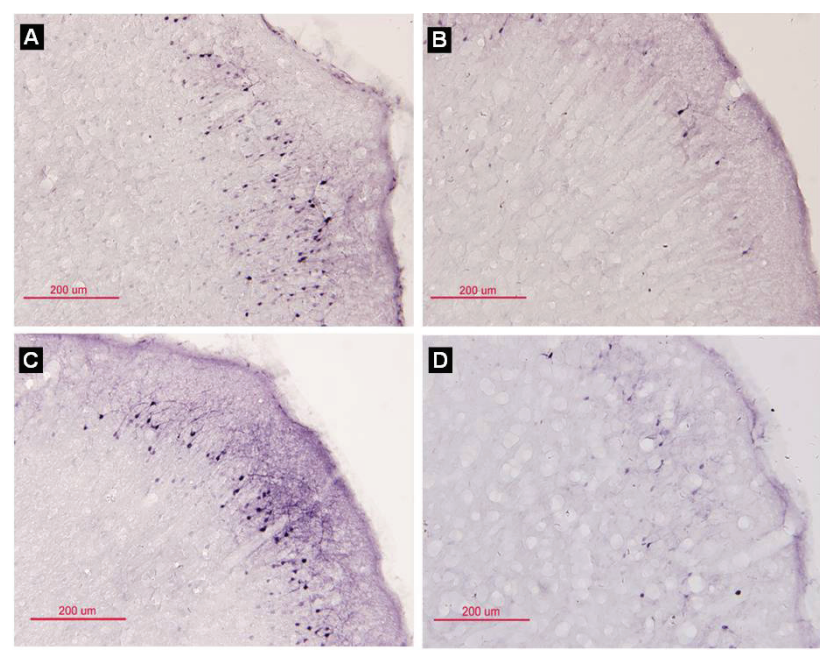

Fig. 5. Representative microphotographs showing the expression of phosphorylated ERK1/2 in the prefrontal cortex in each group. (A) Sham+Veh; (B) ESPS+Veh; (C) ESPS +QUE; (D) QUE+ESPS.

$(\mathrm{P}<0.001)$; but QUE treatment in both pre- and postexposure markedly increased the time spent compared to ESPS-exposed animals $(\mathrm{P}<0.001)$.

\section{pERK1/2 immunoreactive neuronal cells}

There were significant differences between groups on the numbers of pERK1/2 neuronal cells in the three brain regions: $\mathrm{PFC}\left(\mathrm{F}_{3,16}=15.392, \mathrm{P}<0.001\right)$, MAN $\left(\mathrm{F}_{3,16}=12.618, \mathrm{P}<0.001\right)$, and $\mathrm{CG}\left(\mathrm{F}_{3,16}=34.031\right.$, $\mathrm{P}<0.001$ ) (Fig. 4). The numbers of the cells were much lower in ESPS-exposed rats than control animals in all the three brain regions $(\mathrm{P}<0.001)$. Nonetheless, both groups of QUE-treated animals pre- and post-exposed to ESPS had significantly greater numbers of pERK $1 / 2$ cells in MAN and CG compared to ESPS-exposed group $(\mathrm{P}<0.001)$. The number in PFC of rats treated with QUE after ESPS exposure, but not in those treated before 
exposure, was also significantly greater than ESPSexposed rats $(\mathrm{P}<0.001)$ (Fig. 5).

\section{Discussion}

The present study showed that ESPS exposure produced representative anxiety-like behavior, without impairing locomotor function, as evidenced by the fact that ESPS-exposed animals had a significantly decreased time spent and number of entry into open arms in EPM test, but no significant changes in the distance traveled in OF test, indicating the validity of ESPS as an appropriate model for stress-associated anxiety disorders as observed in our previous study (Wang et al. 2008). The current study further demonstrated that ESPS exposure also caused learning and spatial memory impairments, manifested by the strikingly increased latency spent to find the underwater platform and the markedly reduced time spent in the target quadrant when the platform was removed during MWM test. These results are consistent with previous studies, showing cognitive defects in stressed animals (Ryu et al. 2008) and cognitive deteriorations observed in patients with PTSD (Brandes et al. 2002, Golier et al. 2006), suggesting that cognitive impairments may also be an important symptom cluster in PTSD individuals.

Nevertheless, when ESPS-exposed animals were repeatedly administered with QUE, an atypical antipsychotic agent, in either pre- or post-exposure, both decreased EPM parameters were significantly improved. Furthermore, the increased latency to the platform and the reduced time navigated in the target quadrant were reversed to similar levels as observed in control animals. These results suggest that chronic QUE treatment could prevent and protect against anxiety-like behavior and cognitive impairments induced by intensifying stress experience, providing behavioral evidence to support the use of atypical antipsychotic drugs for the treatment of PTSD and other stress-associated anxiety disorders. Our study is also in agreement with clinical studies, showing the effectiveness of QUE in the treatment of PTSD patients with comorbid psychotic symptoms (Hamner et al. 2003, Stathis et al. 2005, Ahearn et al. 2006, Kozaric et al. 2007).

The preventive and protective effects of QUE observed in the present study appear to be mainly achieved through the modulation of related central neurotransmissions. It is well known that, in addition to $\mathrm{D}_{2}$ receptors, QUE also acts on many other receptors, particularly other dopamine and serotonin receptors (Richelson et al. 1999). Several studies have confirmed the associations of altered functions of brain dopaminergic and serotonergic systems with traumatic stress and clinical improvement of anti-PTSD treatment (Horger and Roth 1996, Donnelly 2003, Weiss 2007). Additionally, dysfunction of the hypothalamic-pituitaryadrenal (HPA) axis has been observed in animal models of stress (Liberzon et al. 1997) and PTSD patients (Sapolsky et al. 1984, Yehuda 2001). QUE has been reported to reduce nocturnal urinary cortisol excretion in healthy subjects and steroid-induced mania (Cohrs et al. 2004, Siddiqui et al. 2005). These studies imply that the anti-PTSD efficacy of QUE observed in the current study may also be related to its normalized effects on HPA dysfunction as other atypical antipsychotic agents reported (Zhang et al. 2005).

The present study also found that, while EPSP induced marked decrease in the expression of $\mathrm{pERK} 1 / 2$ neuronal cells in PFC, MAN, and CG, chronic QUE treatment, both before and after exposure to ESPS, significantly elevated the decreased expression of pERK1/2 levels observed in these three brain regions, suggesting that changes in extracellular signal-regulated protein kinase activities of specific brain regions may represent a response to traumatic stress and anti-PTSD treatment. Therefore, the protein kinases, such as pERK1/2 investigated in the present study, could be considered as biomarkers reflecting pathophysiological processing of PTSD and the therapeutic efficacy of antiPTSD treatments, including novel pharmacological therapy, such as atypical antipsychotic drugs. This consideration is also supported by other lines of evidence, confirming that ERK signal system plays a crucial role in protecting against impairments of neuroplasticity and in cellular resilience in the pathophysiology of manicdepressive illness (Manji et al. 2001, Coyle and Duman 2003). The mechanism of involvement of ERK cascade reaction in emotion and cognition is not well understood. However, there are some useful references related to the role of ERK in emotion and cognition. It was reported that B cell lymphoma protein-2 (Bcl-2), Bcl-2 antagonist of cell death (BAD), cAMP response element-binding protein (CREB), and BDNF are downstream targets of ERK and play key roles in neuronal development, survival, and long-term neuronal plasticity (Huang and Reichardt 2001, Dawson and Ginty 2002, Weeber and Sweatt 2002).

In summary, the present study demonstrated that 
the atypical antipsychotic agent QUE has preventive and protective effects against anxiety-like behavior and cognitive impairments induced by traumatic stress in animal model. The study also found that changes in the expression of pERK1/2 levels in specific brain regions are associated with traumatic stress and treatment responses to QUE. These results provide evidence in the support of further evaluation of the effectiveness of atypical antipsychotic treatment of PTSD patients. Detailed mechanisms of ERK signal system in the pathophysiology of stress-related disorders and anti-
PTSD treatment also deserve further investigation.

\section{Conflict of Interest}

There is no conflict of interest.

\section{Acknowledgements}

This work was supported by grants from the Natural Science Foundation of China (No. 30670758/30870886 to QR Tan, No.30700259 to YC Chen, and No. 30670666 to BR Wang), and the 11th Five-Year Project of Military Medicine Foundations (06G096 to Dr. QR Tan).

\section{References}

AHEARN EP, MUSSEY M, JOHNSON C, KROHN A, KRAHN D: Quetiapine as an adjunctive treatment for posttraumatic stress disorder: an 8-week open-label study. Int Clin Psychopharmacol 21: 29-33, 2006.

BRANDES D, BEN-SCHACHAR G, GILBOA A, BONNE O, FREEDMAN S, SHALEV AY: PTSD symptoms and cognitive performance in recent trauma survivors. Psychiatry Res 110: 231-238, 2002.

BREMNER JD: The relationship between cognitive and brain changes in posttraumatic stress disorder. Ann NY Acad Sci 1071: 80-86, 2006.

BROWNING JL, PATEL T, BRANDT PC, YOUNG KA, HOLCOMB LA, HICKS PB: Clozapine and the mitogenactivated protein kinase signal transduction pathway: implications for antipsychotic actions. Biol Psychiatry 57: 617-623, 2007.

CHARLES A, MORGAN III, DORAN A, STEFFIAN G, HAZLETT G, STEVEN M: Stress-induced deficits in working memory and visuo-constructive abilities in special operations soldiers. Biol Psychiatry 60: 722-729, 2006.

COHRS S, POHLMANN K, GUAN Z, JORDAN W, MEIER A, HUETHER G, RÜTHER E, RODENBECK A: Quetiapine reduces nocturnal urinary cortisol excretion in healthy subjects. Psychopharmacology (Berl) 174: 414-420, 2004.

COYLE JT, DUMAN RS: Finding the intracellular signaling pathways affected by mood disorder treatments. Neuron 38: 157-160, 2003.

DAVIDSON JR: Pharmacologic treatment of acute and chronic stress following trauma. J Clin Psychiatry 67: 34-39, 2006.

DAWSON TM, GINTY DD: CREB family transcription factors inhibit neuronal suicide. Nat Med 8: 450-451, 2002.

DONNELLY CL: Pharmacologic treatment approaches for children and adolescents with posttraumatic stress disorder. Child Adolesc Psychiatr Clin N Am 12: 251-269, 2003.

GAO K, MUZINA D, GAJWANI P, CALABRESE JR: Efficacy of typical and atypical antipsychotics for primary and comorbid anxiety symptoms or disorders: a review. J Clin Psychiatry 67: 1327-1340, 2006.

GOLIER JA, HARVEY PD, LEGGE J, YEHUDA R: Memory performance in older trauma survivors: implications for the longitudinal course of PTSD. Ann NY Acad Sci 1071: 54-66, 2006.

HAMNER MB, DEITSCH SE, BRODRICK PS, ULMER HG, LORBERBAUM JP: Quetiapine treatment in patients with posttraumatic stress disorder: an open trial of adjunctive therapy. J Clin Psychopharmacol 23: 15-20, 2003.

HE J, XU HY, YANG Y, RAJAKUMAR D, LI XK, LI XM: The effects of chronic administration of quetiapine on the phencyclidine-induced reference memory impairment and decrease of Bcl-XL/Bax ratio in the posterior cingulate cortex in rats. Behav Brain Res 168: 236-242, 2006.

HORGER BA, ROTH RH: The role of mesoprefrontal dopamine neurons in stress. Crit Rev Neurobiol 10: 395-418, 1996. 
HUANG EJ, REICHARDT LF: Neurotrophins: roles in neuronal development and function. Annu Rev Neurosci 24: 677-736, 2001.

IWAMOTO Y, MORINOBU S, TAKAHASHI T, YAMAWAKI S: Single prolonged stress increases contextual freezing and the expression of glycine transporter 1 and vesicle-associated membrane protein 2mRNA in the hippocampus of rats, Prog Neuropsychopharmacol Biol Psychiatry 31: 642-651, 2007.

KOZARIC-KOVACIC D, PIVAC N: Quetiapine treatment in an open trial in combat-related post-traumatic stress disorder with psychotic features. Int J Neuropsychopharmacol 10: 253-261, 2007.

LESKIN LP, WHITE PM: Attentional networks reveal executive function deficits in posttraumatic stress disorder. Neuropsychology 21: 275-284, 2007.

LIBERZON I, KRSTOV M, YOUNG EA: Stress-restress: effects on ACTH and fast feedback. Psychoneuroendocrinology 22: 443-453, 1997.

LIBERZON I, LOPEZ JF, FLAGEL SB, VAZQUEZ DM, YOUNG EA: Differential regulation of hippocampal glucocorticoid receptors mRNA and fast feedback: relevance to post-traumatic stress disorder. J Neuroendocrinol 11: 11-17, 1999.

LIEBSCH G, MONTKOWSKI A, HOLSBOER F, LANDGRAF R: Behavioural profiles of two Wistar rat lines selectively bred for high or low anxiety-related behaviour. Behav Brain Res 94: 301-310, 1998.

LU XH, DWYER DS: Second-generation antipsychotic drugs, olanzapine, quetiapine, and clozapine enhance neurite outgrowth in PC12 cells via PI3K/AKT, ERK, and pertussis toxin-sensitive pathways. J Mol Neurosci 27: 4364, 2005.

LUO C, XU HY, LI XM: Quetiapine reverses the suppression of hippocampal neurogenesis caused by repeated restraint stress. Brain Res 1063: 32-39, 2005.

LUO XH, RONALD J, BRADLEY, DONARD S, DWYER: Olanzapine produces trophic effects in vitro and stimulates phosphorylation of Akt/PKB, ERK1/2, and the mitogen-activated protein kinase p38. Brain Res 1011: 58-68, 2004.

MANJI HK, DREVETS WC, CHARNEY DS: The cellular neurobiology of depression. Nat Med 7: 541-547, 2001.

PAXINOS G, WATSON C: The Rat Brain in Stereotaxic Coordinates, Academic Press, San Diego, 1998.

PIVAC N, KOZARI-KOVACI D: Pharmacotherapy of treatment-resistant combat-related posttraumatic stress disorder with psychotic features. Croat Med J 47: 440-451, 2006.

QI X, LIN W, WANG D, PAN Y, WANG W, SUN M: A role for the extracellular signal-regulated kinase signal pathway in depressive-like behavior. Behav Brain Res 199: 203-209, 2009.

RASMUSSEN K: Creating more effective antidepressants:clues from the clinic. Drug Discov Today 11: 623-631, 2006.

RAUCH SA, GRUNFELD TE, YADIN E, CAHILL SP, HEMBREE E, FOA EB: Changes in reported physical health symptoms and social function with prolonged exposure therapy for chronic posttraumatic stress disorder. Depress Anxiety 26: 732-738, 2009.

RICHELSON E: Receptor pharmacology of neuroleptics: relation to clinical effects. J Clin Psychiatry 60 (Suppl 10): 514, 1999.

RYU J, FUTAI K, FELIU M, WEINBERG R, SHENG M: Constitutively active Rap2 transgenic mice display fewer dendritic spines, reduced extracellular signal-regulated kinase signaling, enhanced long-term depression, and impaired spatial. J Neurosci 28: 8178-8188, 2008.

SAPOLSKY RM, KREY LC, MCEWEN BS: Glucocorticoid-sensitive hippocampal neurons are involved in terminating the adrenocortical stress response. Proc Natl Acad Sci USA 81: 6174-6177, 1984.

SIDDIQUI Z, RAMASWAMY S, PETTY F: Quetiapine therapy for corticosteroid-induced mania. Can J Psychiatry 50: 77-78, 2005.

STATHIS S, MARTIN G, MCKENNA JG: A preliminary case series on the use of quetiapine for posttraumatic stress disorder in juveniles within a youth detention center. J Clin Psychopharmacol 25: 539-454, 2005.

TAKAHASHI T, MORINOBU S, IWAMOTO Y, YAMAWAKI S: Effect of paroxetine on enhanced contextual fear induced by single prolonged stress in rats. Psychopharmacology 189: 165-173, 2006.

WANG W, LIU Y, ZHENG H, WANG HN, JIN X, CHEN YC, ZHENG LN, LUO XX, TAN QR: A modified singleprolonged stress model for post-traumatic stress disorder. Neurosci Lett 441: 237-241, 2008. 
WANG X, ZHANG XJ, TAN QR, JU G, WANG BR: Different signaling molecules responsible for IL-1beta-induced oxytocinergic and vasopressinergic neuron activation in the hypothalamic paraventricular nucleus of the rat. Neurochem Int 48: 312-317, 2006.

WEEBER EJ, SWEATT JD: Molecular neurobiology of human cognition. Neuron 33: 845-848, 2002.

WEISS SJ: Neurobiological alterations associated with traumatic stress. Perspect Psychiatry Care 43: 114-122, 2007.

YEHUDA R: Biology of posttraumatic stress disorder. J Clin Psychiat 62 (Suppl): 41-46, 2001.

ZHANG XY, ZHOU DF, CAO LY, WU GY, SHEN YC: Cortisol and cytokines in chronic and treatment-resistant patients with schizophrenia: association with psychopathology and response to antipsychotics. Neuropsychopharmacology 8: 1532-1538, 2005.

ZHENG G, CHEN YM, ZHANG XP, CAI TJ, LIU MC: Acute cold exposure and rewarming enhanced spatial memory and activated the MAPK cascades in the rat brain. Brain Res 1239: 171-180, 2008. 